\title{
Temperature profile of ex-vivo organs during radio frequency thermal ablation by fiber Bragg gratings
}

Giovanna Palumbo

Agostino Iadicicco

Daniele Tosi

Paolo Verze

Nicola Carlomagno

Vincenzo Tammaro

Juliet Ippolito

Stefania Campopiano 


\title{
Temperature profile of ex-vivo organs during radio frequency thermal ablation by fiber Bragg gratings
}

\author{
Giovanna Palumbo, ${ }^{a}$ Agostino ladicicco, ${ }^{a}$ Daniele Tosi, ${ }^{b}$ Paolo Verze,${ }^{c}$ Nicola Carlomagno, ${ }^{d}$ \\ Vincenzo Tammaro, ${ }^{\mathrm{d}}$ Juliet Ippolito, ${ }^{\mathrm{c}}$ and Stefania Campopiano ${ }^{\mathrm{a}, *}$ \\ aUniversity of Naples "Parthenope", Centro Direzionale Isola C4, Naples 80143, Italy \\ ${ }^{b}$ Nazarbayev University, School of Engineering, 53 Kabanbay Batyz Avenue, Astana 010000, Kazakhstan \\ ${ }^{\mathrm{C}}$ Azienda Ospedaliera Universitaria Federico II of Naples, Urology Unit, Via S. Pansini 5, Naples 80131, Italy \\ ${ }^{\mathrm{d} A z i e n d a}$ Ospedaliera Universitaria Federico II of Naples, General Surgery and Transplant Unit, Via S. Pansini 5, Naples 80131, Italy
}

\begin{abstract}
We report on the integration of fiber optic sensors with commercial medical instrumentation for temperature monitoring during radio frequency ablation for tumor treatment. A suitable configuration with five fiber Bragg grating sensors bonded to a bipolar radio frequency (RF) probe has been developed to monitor the area under treatment. A series of experiments were conducted on ex-vivo animal kidney and liver and the results confirm that we were able to make a multipoint measurement and to develop a real-time temperature profile of the area, with a temperature resolution of $0.1^{\circ} \mathrm{C}$ and a spatial resolution of $5 \mathrm{~mm}$ during a series of different and consecutive RF discharges. () The Authors. Published by SPIE under a Creative Commons Attribution 3.0 Unported License. Distribution or reproduction of this work in whole or in part requires full attribution of the original publication, including its DOI. [DOI: 10.1117/1.JBO.21.11.117003]
\end{abstract}

Keywords: fiber optic sensors; fiber Bragg gratings; temperature monitoring; radio frequency ablation; thermotherapy.

Paper 160537R received Aug. 6, 2016; accepted for publication Oct. 19, 2016; published online Nov. 15, 2016.

\section{Introduction}

Since its acceptance into clinical practice in 1990s, thermal ablation has been widely used as a viable minimally invasive treatment for kidney ${ }^{1}$ and liver ${ }^{2}$ tumor therapy and is fast becoming the preferred therapy over the standard invasive procedure. In the liver and kidney organs in particular, each and every functional unit - the hepatic acinus in the liver and the nephron in the kidney - that can be saved, counts in a surgeon's attempt to preserve as much functional organ tissue as possible in their oncological patients.

Among other thermal ablation treatments, radio frequency ablation (RFA) is considered to be the most effective percutaneous techniques for patients with primary or metastatic liver cancer (hepatocellular carcinoma) ${ }^{3}$ and primary renal cancer, as specified in hepatology ${ }^{4}$ and urological ${ }^{5}$ guidelines. Indeed, as primary liver tumors are often soft and encapsulated, RFA allows for effective heat disbursement and retention while in kidney cancer, minimally invasive RFA therapy has been found to postpone kidney failure and prolong kidney function in patients with multiple or hereditary renal cell carcinoma. ${ }^{3}$ Today, RFA is considered a useful option for patients who are deemed to be nonoperative candidates or who may have a solitary kidney, multiple medical problems, or unresectable tumors. Due to its cauterizing effect, RFA therapy combined with chemoembolization, can selectively block blood flow to a tumor thereby providing more effective treatment for larger tumors. ${ }^{6}$ Its "heat sink effect" results in little collateral damage during treatments situated near vital structures and serves to preserve the vessels near the tumor treatment area.

RFA application strictly relies on the thermal shock to the cell, therefore, the precision of its application is fundamental

*Address all correspondence to: Stefania Campopiano, E-mail: campopiano@ uniparthenope.it for two reasons: efficacy and safety. Its efficacy lies in the fact that only the cancerous cells exposed to the thermal discharges are treated, and its safety lies in the fact that healthy cell tissues do not need to be unnecessarily destroyed. Therefore, accurate measuring of tissue temperature in the radio frequency (RF)-applied region represents a valid feedback for the electronic control unit and for the operating medical team. ${ }^{7}$

In this context, several external methods have been explored for accurate temperature measurements during thermal ablation treatments: external thermocouples, computed tomography (CT)-imaging, ultrasound, and magnetic resonance imaging (MRI). CT-thermometry is noninvasive but has the dual disadvantages of low reproducibility and resolution and also requires precise calibration. Ultrasound-based techniques, while being noninvasive and giving better spatial resolution, can, however, be distorted by physiological movements. For example, MRIthermometry can lead to measurement errors and is more expensive compared with other thermal monitoring technologies. ${ }^{7,8}$ Finally, even though thermocouples are often invasive and have poor metrological properties (slow response and heat sink effect) to date, they are the most used approach of RFA instrumentations. ${ }^{9}$ Indeed, in the last generation of commercial RFA devices, temperature measurement is typically provided by means of 1 to 5 mini-thermocouple sensors mounted on the device tip(s), or by measuring the tissue impedance, which allows an indirect temperature estimation. In this way, temperature is directly or indirectly measured only in the region reached by the RF electrodes. Moreover, the temperature gradient versus the distance from the electrodes is estimated by means of the tissue thermal impedance and/or by the expertise of the operator. This ensures maintaining appropriate error margin with consequent enlargement of the thermally treated tissue region.

An alternative technological platform is based on fiber optic sensors (FOSs), which show great potential in the biomedical applications field. FOSs are small in size, light enough in weight 
(a)
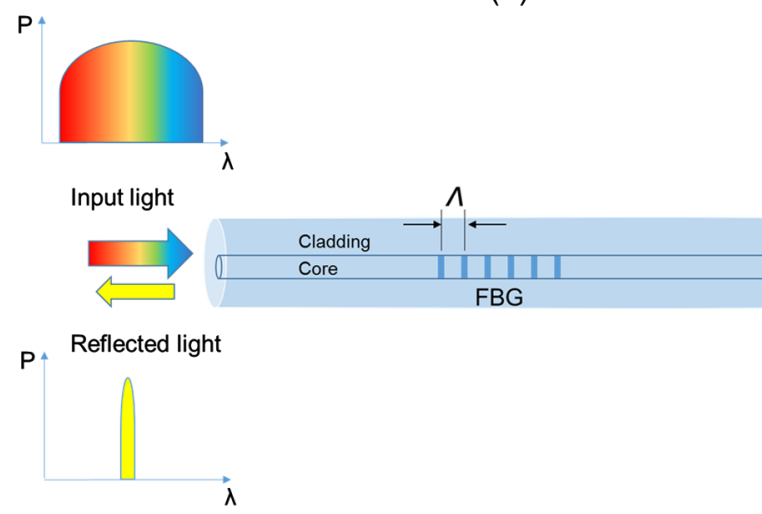

(b)

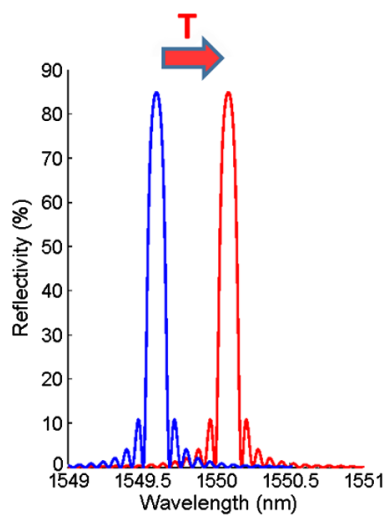

Fig. 1 FBG: (a) principle of operation and (b) wavelength shift when a temperature increase of $60^{\circ} \mathrm{C}$ is applied.

to meet minimally invasive requirements, biocompatible (in compliance with International Organization for StandardizationISO-standard 10993, commonly referred to by the Food and Drug Administration and the European Medicines AgencyEMA), nontoxic, chemically inert, immune to the induced-RF field during ablation, and, furthermore, allow for remote operation. ${ }^{10}$ Moreover, FOSs can be easily embedded into surgical instruments, such as catheters and needles, and guided to a precise target during surgery. ${ }^{11,12}$

Among FOSs, fiber Bragg gratings (FBGs), whose sensing potentials have been widely demonstrated over the last decade in a number of sensing fields ranging from aerospace to civil engineering and environmental monitoring, ${ }^{13,14}$ are fast becoming increasingly popular in the medical sensing field ${ }^{15}$ as well. For example, FBGs have been experimented with for monitoring bone decalcification, ${ }^{16}$ pressure monitoring, ${ }^{17,18}$ controlling respiratory movements, ${ }^{19}$ as well as specific sensors developed for $\mathrm{pH}$ and refractive index of liquids. ${ }^{20-22}$ FBGs have also been used as temperature sensors, ${ }^{23}$ as well as in vibration measurement during treatment of atrial fibrillation. ${ }^{24-26}$ Moreover, FBG technology has been used in experiments for thermal measurements during $\mathrm{RF}$ and/or laser ablation therapy. ${ }^{8,27,28}$ Tosi et al. ${ }^{29}$ conducted experiments on thermal monitoring during RFA of liver tumors using an array of five draw-tower FBGs fabricated on an ormoceramic bend-insentive fiber as well as on a distributed FOS using chirped FBGs. ${ }^{27,30,31}$ However, to the best of our knowledge, thermal monitoring based on FOSs has only been applied to hepatic and pancreatic tissues based upon a single-step RFA, laser, and microwave ablation. ${ }^{32}$

In this paper, we propose the use of FBG sensors array for measuring of the spatial temperature profile during RFA thermal application $^{33}$ to effectively study, monitor, and accurately measure heat propagation inside animal organ tissues. The results of our experiments lead us to believe that a similar approach could be applied to give a more controlled range of heat dispersion and leave a larger safety margin around the perimeter of the tumor section being removed. This approach has been validated by a wide experimental analysis of multistep RFA treatments on ex-vivo animal liver and kidney organs as reported herein.

\section{Design of a Sensorized Radio Frequency Probe}

Our first step was to design and fabricate an RF probe that was able to measure temperature gradient. To this aim, a commercial
RF probe was sensorized with several FBG sensors properly packaged in a loose metallic tube. The FBG-equipped RF probe was then applied on ex-vivo animal liver and kidneys. The different components of our setup will be subsequently illustrated.

\subsection{Fiber Bragg Gratings Principle of Operation}

The uniform FBG is an optical sensor fabricated within the core of a standard, single-mode optical fiber. It consists of a segment of optical fiber along which a spatially periodic modulation of the core refractive index is permanently inscribed. The fabrication process is possible due to the photosensitivity property of the doped silica glass fiber core. Normally, a germanium dopant is used. Figure 1(a) shows the primary structure of an FBG with its typical length of circa 1 to $20 \mathrm{~mm}$.

The FBG principle of operation is based on the coupling between the forward and the backward propagating core modes. When light propagates through a fiber with a Bragg grating, a reflection phenomenon for a narrow range of wavelengths occurs while other wavelengths are transmitted. The reflected wavelength, centered at a specific value, is called a Bragg wavelength $\lambda_{B}$ [Fig. 1(a)]. ${ }^{34-36}$

The Bragg wavelength satisfies the homonymous condition given by

$\lambda_{B}=2 n_{\mathrm{eff}} \Lambda$,

where $\Lambda$ is the grating period and $n_{\text {eff }}$ is the effective refractive index of the core propagation mode. The working principle commonly used in FBG sensors is based on $\lambda_{B}$ shift due to a variation of the spatial period of the grating and to an effective refractive index variation of the core. Consequently, the external physical parameters, temperature and strain, can be detected through the measurement of the reflected wavelength.

Strain and temperature contributions can be separated through the implementation of specific sensing configurations. For our purposes, the FBG has been employed in a "strain-free" configuration in order to consider only the temperature contribution. In this hypothesis, the influence of external temperature on the wavelength shift is dependent on: (i) the thermal expansion coefficient, i.e., the expansion of the grating and (ii) the thermo-optic coefficient, i.e., the change in the refractive index. The change of the FBG central wavelength is given as follows: ${ }^{26,37}$ 
(a)

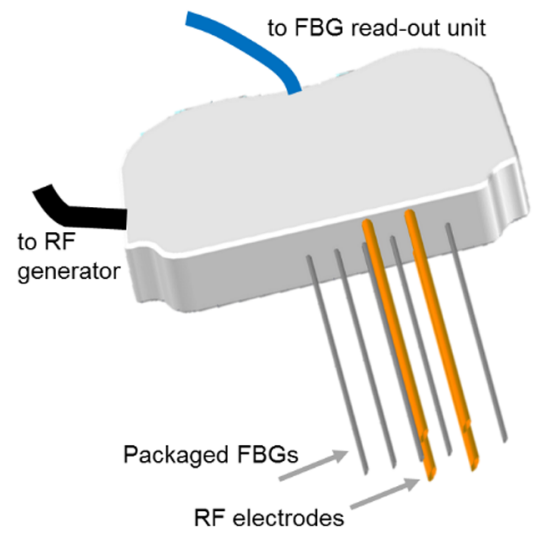

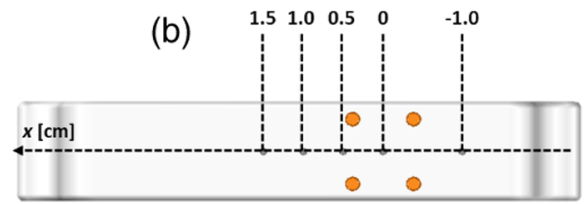

Bottom view

(c)

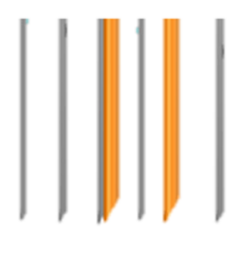

Side view

Fig. 2 Schematic of the RF probe: (a) perspective, (b) bottom, and (c) side view.

$\frac{1}{\lambda_{B}} \cdot \frac{\mathrm{d} \lambda_{B}}{\mathrm{~d} T}=\frac{1}{n_{\mathrm{eff}}} \cdot \frac{\mathrm{d} n_{\mathrm{eff}}}{\mathrm{d} T}+\frac{1}{\Lambda} \cdot \frac{\mathrm{d} \Lambda}{\mathrm{d} T}$,

where $\left[\left(1 / n_{\text {eff }}\right) \cdot\left(\mathrm{d} n_{\text {eff }} / \mathrm{d} T\right)\right]=\xi$ is the thermal-optical coefficient and $[(1 / \Lambda) \cdot(\mathrm{d} \Lambda / \mathrm{d} T)]=\alpha$ is the thermal expansion coefficient of the FBG. Therefore, the Bragg wavelength change due to temperature can be written as

$\frac{\mathrm{d} \lambda_{B}}{\lambda_{B}}=(\xi+\alpha) \mathrm{d} T$.

It is possible to simplify the equation introducing the thermal sensitivity coefficient $S_{T}=(\xi+\alpha)$. Consequently, Eq. (3) can be written as

$\frac{\mathrm{d} \lambda_{B}}{\lambda_{B}}=S_{T} \mathrm{~d} T$.

Thus, every temperature variation will cause a change in the pitch of the grating and in the effective refractive index causing a shift in the Bragg wavelength according to Eq. (4). This behavior is shown in Fig. 1(b).

\subsection{Setup for Radio Frequency Ablation with Temperature Measurements}

A commercial RF probe made available at the General Surgery and Transplant Unit of the University Hospital Azienda Ospedaliera Universitaria (AOU) "Federico II," was equipped with five properly packaged FBG sensors. A schematic view of the modified probe is plotted in Fig. 2.

The pristine RF probe is a laparoscopic bipolar RF device for bloodless laparoscopic liver resection, currently employed by the general surgeons participating in our experiment. The device consists of two pairs of opposing electrodes with active ends of $6 \mathrm{~cm}$ in length ${ }^{37,38}$ that are spaced forming a square of side $6.0 \mathrm{~mm}$, schematically plotted in Fig. 2(a). The device is designed to provide RF energy to be used for coagulation and ablation of soft tissue and is indicated for use in percutaneous, laparoscopic, or intraoperative procedures. The RF probe is powered by a $500-\mathrm{kHz}$ generator, which produces up to $250 \mathrm{~W}$ of RF power. The system also allows the measurement of generator output, temperature, impedance, and time through an LCD. ${ }^{39}$
In this work, the RF probe was properly modified to hold five FBGs spatially distributed along the $x$-axis. Figures 2(b) and 2(c) show a bottom and side view of the probe, respectively, where the $x$-axis and optical sensors distribution are evident. Four FBGs are positioned at $x=0 \mathrm{~cm}, x=0.5 \mathrm{~cm}, x=$ $1.0 \mathrm{~cm}$, and $x=1.5 \mathrm{~cm}$ in order to measure a temperature profile starting from the electrode's center. The last FBG was fixed at $-1.0 \mathrm{~cm}$ in order to observe the symmetry of the temperature profile.

Here, FBGs written in standard SMF-28e fiber with a $20-\mu$ m-thick polyimide coating were used. The FBG sensors were properly inserted in a metallic package in order to permit easy insertion and extraction of the modified probe in the ex-vivo organs, as schematically plotted in Fig. 3(a). The packages were realized by means of medical needles (Chiba needles Coloplast, SS7201) with an inner hole of $700 \mu \mathrm{m}$. The ends of the needles were then enclosed with a small cap of cyanoacrylate adhesive in order to ensure FBGs strain-free operations. Finally, the Chiba needles were fixed to the probe so that their tips were in the same position as those of the electrodes [see Fig. 2(c)]. All FBGs were $5.0 \mathrm{~mm}$ in length and positioned at the bottom of the needles in order to keep the sensing region as close as possible to the electrodes' ends. In Fig. 3(b), a photograph of the modified RF probe is also shown.

A commercial optoelectronic unit was used to measure the FBGs reflected signal, which included a tunable laser operating in the range of 1500 to $1600 \mathrm{~nm}$ that is equipped with eight optical channels allowing for simultaneous measurement of a large number of sensors (up to 25 for each channel). The detector measured the Bragg wavelengths with a resolution of $1 \mathrm{pm}$ with a minimum sampling frequency of 1 sample/s.

The bare FBGs were thermally characterized in a controlled oven up to $120^{\circ} \mathrm{C}$, and the final configuration, i.e., the packaged FBG sensors attached to the RF probe, was successively thermally characterized in hot water. The water element was chosen to create an environment similar to the experiments to follow, and as a result of this choice, its temperature was limited to the boiling point and evaporation. Temperature sensitivity characterization was carried out using PT- $100\left(0.03^{\circ} \mathrm{C}\right.$ accuracy $)$ as a reference in the temperature range of $23^{\circ} \mathrm{C}$ to $72^{\circ} \mathrm{C}$. Figure 4 (a) shows the relative Bragg wavelength shifts of all sensors versus the water temperature exhibiting a linear behavior. The FBGs show that a wavelength shift between 10.5 and $10.7 \mathrm{pm} /{ }^{\circ} \mathrm{C}$ (with a standard deviation less than $1.4 \mathrm{pm}$ ) was 
(a)

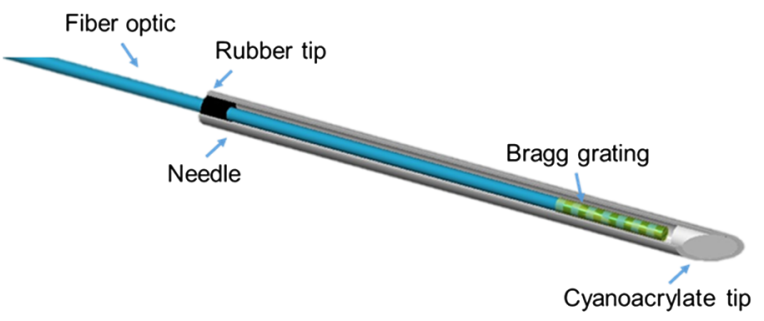

(b)

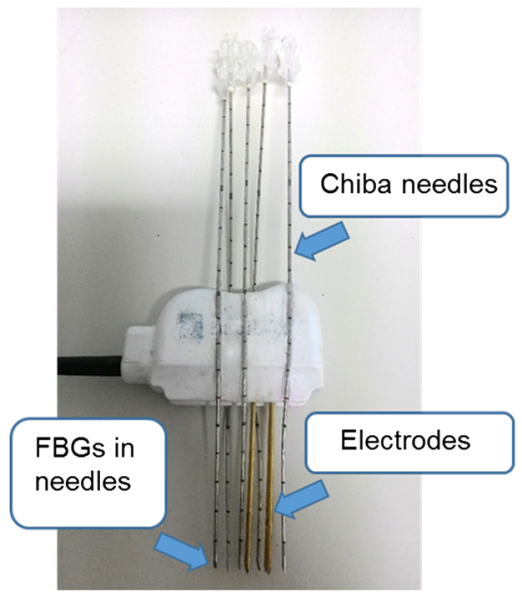

Fig. 3 (a) Schematic of the FBG package and (b) image of the modified RF probe.

(a)

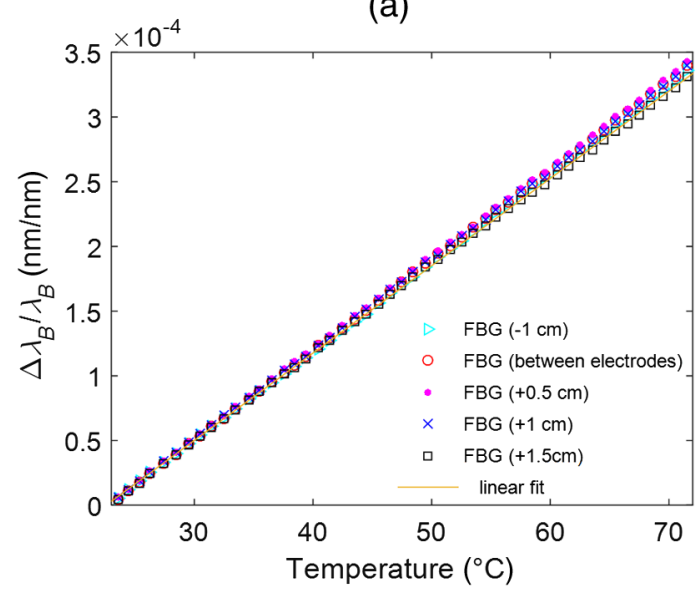

(b)

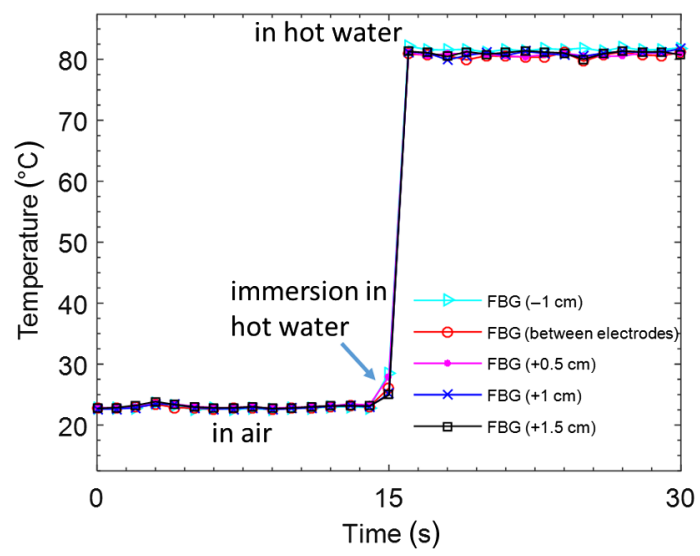

Fig. 4 (a) FBGs relative wavelength shifts versus water temperature $T$ and (b) temperatures read by the FBGs due to the probe immersing in hot water.

found, with a corresponding thermal sensitivity coefficient between $6.4 \times 10^{-6 \circ} \mathrm{C}^{-1}$ and $6.7 \times 10^{-6{ }^{\circ}} \mathrm{C}^{-1}$. Therefore, with the optoelectronic setup, temperature used measurements with a resolution of $0.1^{\circ} \mathrm{C}$ were made possible.

Moreover, in order to investigate the response time of the packaged FBGs, the FBG-equipped probe was manually immersed in hot water and the resulting temperatures as read by the FBGs were plotted in Fig. 4(b). As can be seen, the FBGs exhibit a fast temperature response time (less than $1 \mathrm{~s}$ ), which was deemed sufficient for our purposes. Unfortunately, the exact temporal temperature response evaluation was not possible because the optical interrogator-sampling rate was limited to 1 sample/s.

\section{Results and Discussion}

Experiments were carried on ex-vivo liver and kidney animal tissues at a dry laboratory of the Urology Unit of the University Hospital AOU Federico II, in agreement with EU protocols. In particular, our research team focused on the analysis of the temperature recorded by FBG sensors during RFA treatment, which consisted of consecutive insertions of the sensorized RF probe, each emitting a series of RF discharges.
The RFA electrodes, as well as the five FBGs, were inserted approximately $3-\mathrm{cm}$ deep into the organs.

Figure 5(a) reports the temperature variations during the thermoablation treatment that consisted of two consecutive discharges at about $30 \mathrm{~s}$ and shortly after $2 \mathrm{~min}$, respectively, on the ex-vivo liver. The test was carried out on a liver sample prewarmed at $40^{\circ} \mathrm{C}$ in order to bring the sample as close as possible to body temperature. The two discharges are clearly visible in the reported temperature profile. As expected, the maximum temperature was recorded by the FBG positioned in the center of the electrodes (red curve) while the lowest temperature (about $45^{\circ} \mathrm{C}$ ) is recorded by the $\mathrm{FBG}$ at a distance of $1.5 \mathrm{~cm}$ from the center of discharges (black curve). The sensor in the center of the electrodes reached maximum temperatures of $102.3^{\circ} \mathrm{C}$ and $97.7^{\circ} \mathrm{C}$ during the first and second discharges, respectively. Also, at the first discharge, a maximum temperature of about $80^{\circ} \mathrm{C}$ was recorded at a distance of $0.5 \mathrm{~cm}$ from the electrodes, while temperatures of $53{ }^{\circ} \mathrm{C}$ and of $56^{\circ} \mathrm{C}$ at -1 and $+1 \mathrm{~cm}$ were measured, respectively. Finally, at a distance of $1.5 \mathrm{~cm}$ from the electrodes, a temperature value of circa $50^{\circ}$ $\mathrm{C}$ is recorded. The second discharge shows a comparable temperature behavior. It can further be noted that when leaving the 
(a)

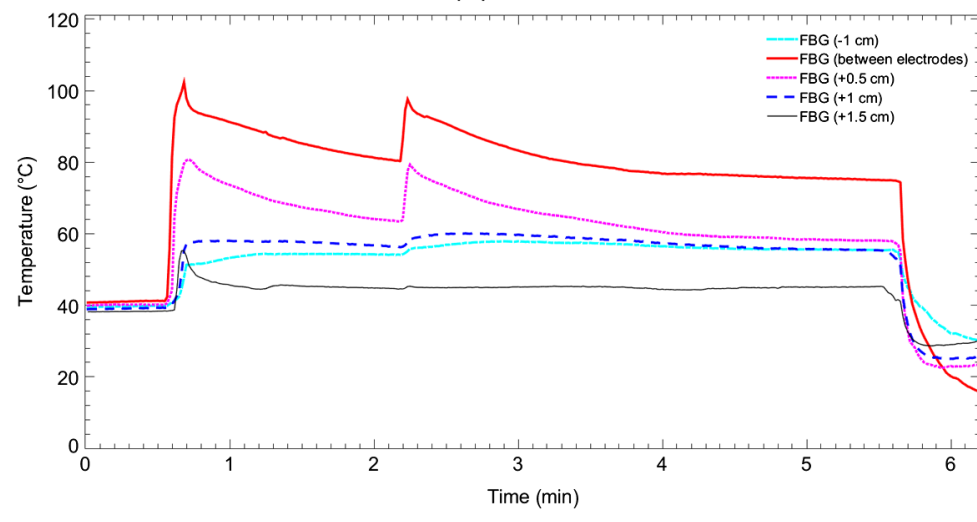

(b)

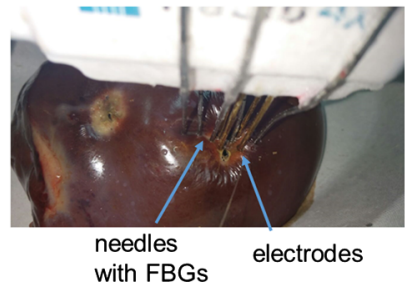

Fig. 5 (a) FBGs temperature variation and (b) insertion of RF probe in liver.

electrodes in the organ, the temperature around the probe (red line) remained higher than that of the surrounding area (black line). Finally, it was possible to distinguish the rapid return to room temperature (about $23^{\circ} \mathrm{C}$ ) once the probe was extracted from the organ. It is worth noting that we conducted several experiments similar to this one, and due to the inhomogeneity of the organ tissue, which is dependent on the electrical properties (impedance) and their evolution throughout RFA, large variations in the self-same tissue under thermal ablation treatment have been noted. ${ }^{40}$ This inherent tissue inhomogeneity introduces asymmetries in heat propagation. Furthermore, after the first discharge, the characteristic of the tissue changes resulting in a different temperature response between each discharge (indeed the FBG at $1.5 \mathrm{~cm}$ shows a peak during the first discharge whereas during the second one it exhibits a small temperature variation).

According to a recent and comprehensive model of heat propagation in liver tissue, it is possible to observe the heat diffusion at a certain distance from the electrodes. ${ }^{40}$ Even if this referenced model does not completely fit our setup due to its being drawn for one electrode and 15- to $30-\mathrm{W}$ RF power, when we used a different applicator with four electrodes and high power with a very short pulse, ${ }^{40}$ the temperature pattern obtained during our experiments was found to be compatible with the work in Ref. 29.

Figure 5(b) contains a photo of the liver during an insertion.

In order to better observe the temperature profile as a function of spatial distribution as well as of time, temperature space-time maps are presented and discussed.

Figures 6(a) and 6(b) contain temperature maps during two different tests in the ex-vivo liver (different probe insertions in different tissue locations) with two and three RF discharges, respectively. In these figures, temperature is reported by a color map versus spatial sensor position (vertical axis in the graph) and time (horizontal axis). It is worth noting that the temperature data versus spatial position were achieved by a piecewise linear interpolation of the experimental data points. For clarity, the sensors' position is also indicated in Fig. 6(a). In the same figure, we also report on a series of isothermal curves at different temperature values by means of dashed lines. As can be seen, the spatial temperature profile is not symmetric with respect to the RF probe, most likely due to tissue inhomogeneity, which confirms that the direct temperature measurements are a useful tool for decreasing the ablated tissue region. Moreover, it is clear that the isothermal curves move far from the RF probe center as a function of the number (a)

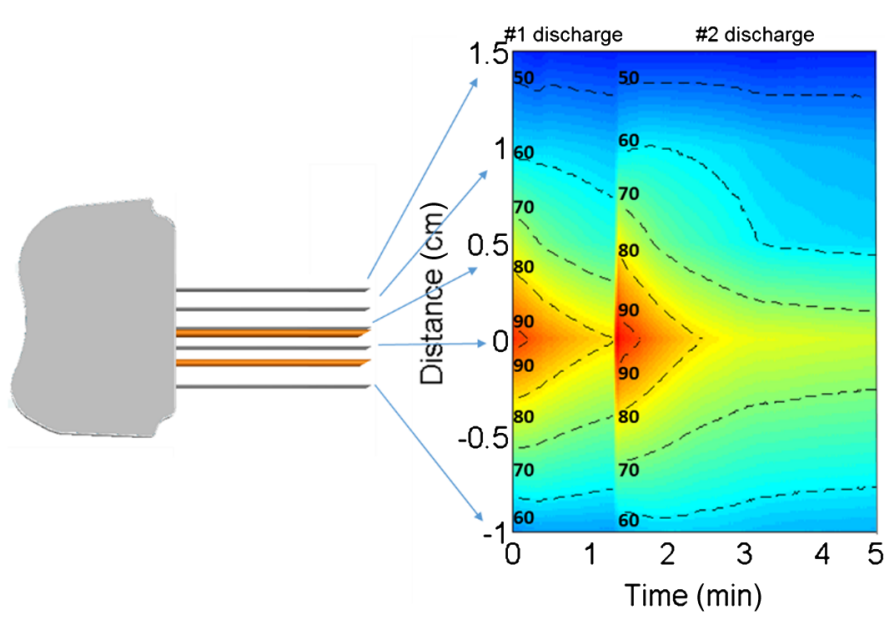

(b)

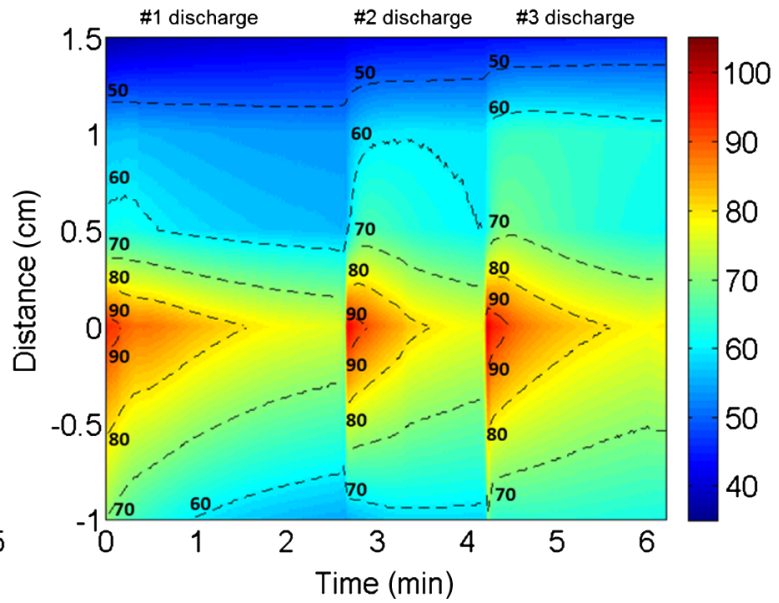

Fig. 6 (a) Temperature profile during first insertion in liver and (b) during second insertion in liver. 
(a)

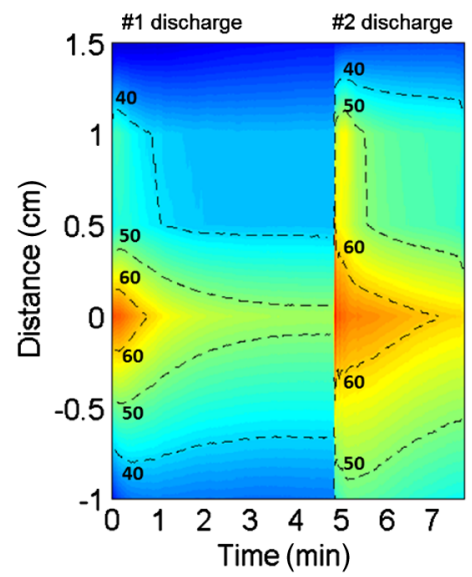

(b)

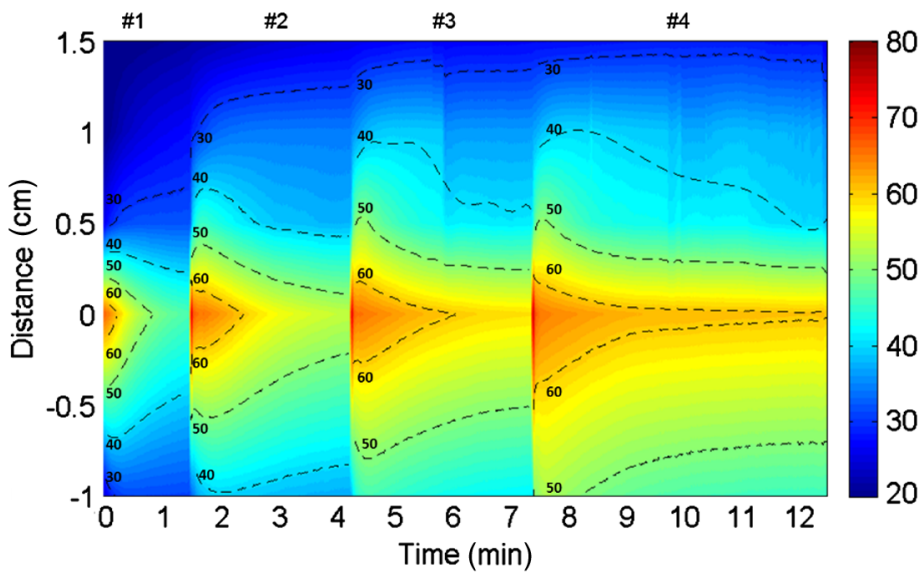

Fig. 7 (a) Temperature profile during first insertion in kidney and (b) during second insertion in kidney.

of discharges, since the organ tissue is not able to release the heat quickly. In addition, it should be considered that we experimented on a small (a few centimeters) piece of liver, hence, the temperature profile shows values slightly higher than expected.

Similar experiments were also carried out on ex-vivo bovine kidney (Fig. 7), where two separate insertions in the organ are presented. During the first insertion with the two discharges, Fig. 7(a) shows that the temperature of the ablated area decreases with an increase in distance from the target area. Similar results are shown for the second kidney insertion [Fig. 7(b)]. In the second insertion, where four discharges were made, the temperature profile is in accord with that observed in the previous insertion, the temperature profile decreases as the distance from the peak ablation increases, and spatially increases as a function of the discharges number.

\section{Conclusions}

In our experiments on ex-vivo animal liver and kidney tissues, we have demonstrated the integration of fiber optic technology with a commercial medical instrumentation based on RFA for tumor ablation, which enabled a real-time temperature profile monitoring of medical thermal treatment. In both series of tests, as reported, we were able to successfully identify and measure the temperature profile near the RF probe with five different sensors with a spatial resolution of $5 \mathrm{~mm}$. Thanks to the FBGs' fast response time, we were able to monitor temperature with a resolution of $0.1^{\circ} \mathrm{C}$ during different and consecutive discharges. This mapping of the tissue temperature enabled us to follow the movement of the RF probe and create a temperature profile in accordance with the different positions of the probe during the thermoablation treatment. The proposed FBG-based solution is able to distinguish between the different discharges and to measure the temperature profile for each of them, where the temperature decreases with each increase in distance. This profiling is not possible with conventional thermal ablation technology currently in use.

The ability to perform real-time temperature profile monitoring of the thermal ablation treatment area has several potential clinical implications that are worth mentioning. First, the existence of high-quality and precise measurements would allow for an optimization of the efficacy-safety pattern of the RFA procedure. For these reasons, FOSs have the potential to clearly delineate the ideal tissue area to be ablated before surgical procedure, which should be the real target of the thermal probe, thereby allowing the surgeon to necrotize as many tumor cells as necessary and at the same time spare as many healthy cells as possible, thus perfectly fulfilling the requirements for a minimally invasive procedure. For this purpose, a better integration of the imaging study of the tumor lesion with the preoperative plan of the thermal application is strongly advised in order to optimize the ideal area to be treated. At the same time, real-time temperature monitoring with FOSs along the surgical target area perimeter would allow for verification that an optimal presurgical map was achieved once the procedure is completed. The clinical utility of the proposed thermal ablation treatment incorporating fiber optic sensors leads to the possibility of perfectly identifying the thermally treated area in order to minimize the risk of positive surgical margins whereby the cancerous cells come right out to the edge of the removed tissue.

The strength of the present research is the novelty of a biomedical application of a temperature check using FOSs that can ensure both high precision and reliability of the measurements. Furthermore, the reproducibility of the sensors' use on both hepatic and renal tissue as reported in our experiments paves the way for application of this technology in a wide range of surgical procedures. Future studies will be made to confirm the reliability of the FOSs in a real-time temperature profile monitoring of thermal ablation treatment area on human tissues in an ex-vivo experimental setting.

\section{Acknowledgments}

This work was supported by the University of Naples "Parthenope" through the "Progetto locale."

\section{References}

1. H. Higgins and D. L. Berger, "RFA for liver tumors: does it really work?" Oncologist 11(7), 801-808 (2006).

2. K. G. Kwan and E. D. Matsumoto, "Radiofrequency ablation and cryoablation of renal tumours," Curr. Oncol. 14(1), 34-38 (2007).

3. "NIH CC: radiofrequency thermal ablation as tumor therapy," http:// www.cc.nih.gov/drd/tumortherapy.html (June 2009).

4. The European Association for the Study of liver and European Organisation for Research and Treatment of Cancer, "EASL-EORTC clinical practice guidelines: management of hepatocellular carcinoma," J. Hepatol. 56(4), 908-943, (2012). 
5. B. Ljungberg et al., "EAU guidelines on renal cell carcinoma: 2014 update," Eur. Urol. 67(5), 913-924 (2015).

6. S. A. Curley, "Radiofrequency ablation of malignant liver tumors," Ann. Surg. Oncol. 10(4), 338-347 (2003).

7. E. M. Knavel and C. L. Brace, "Tumor ablation: common modalities and general practices," Tech. Vasc. Interv. Radiol. 16(4), 192-200 (2013).

8. P. Saccomandi et al., "Techniques for temperature monitoring during laser-induced thermotherapy: an overview," Int. J. Hyperth. 29(7), 609-619 (2013).

9. J. P. McGahan and G. D. Dodd, "Radiofrequency ablation of the liver," Am. J. Roentgenol. 176(1), 3-16 (2001).

10. E. Al-Fakih, N. A. A. Osman, and F. R. M. Adikan, "The use of fiber Bragg grating sensors in biomechanics and rehabilitation applications: the state-of-the-art and ongoing research topics," Sensors 12(10), 12890-12926 (2012).

11. D. R. Biswas, "Optical fiber coatings for biomedical applications," Opt. Eng. 31(7), 1400-1403 (1992)

12. P. Roriz et al., "Review of fiber-optic pressure sensors for biomedical and biomechanical applications," J. Biomed. Opt. 18(5), 050903 (2013).

13. A. Iadicicco et al., "Strain measurements of a multilayer panel via Fiber Bragg gratings as novel approach for deflection monitoring of tracking particle detectors," Proc. SPIE 9506, 95061L (2015).

14. A. Iadicicco et al., "Deflection monitoring method using fiber Bragg gratings applied to tracking particle detectors," IEEE Photonics J. 6(6), 1-10 (2014)

15. V. Mishra et al., "Fiber grating sensors in medicine: current and emerging applications," Sens. Actuators A Phys. 167(2), 279-290 (2011).

16. P. Roriz et al., "From conventional sensors to fibre optic sensors for strain and force measurements in biomechanics applications: a review," J. Biomech. 47(6), 1251-1261 (2014).

17. Q. Yu and X. Zhou, "Pressure sensor based on the fiber-optic extrinsic Fabry-Perot interferometer," Photonic Sens. 1(1), 72-83 (2011).

18. S. Poeggel et al., "Optical fibre pressure sensors in medical applications," Sensors 15(7), 17115-17148 (2015).

19. J. Witt et al., "Medical textiles with embedded fiber optic sensors for monitoring of respiratory movement," IEEE Sens. J. 12(1), 246-254 (2012).

20. J. Lin, "Recent development and applications of optical and fiber-optic pH sensors," Trends Anal. Chem. 19(9), 541-552 (2000).

21. A. Iadicicco et al., "Micro-structured fiber Bragg gratings: optimization of the fabrication process," Opt. Express 15(23), 15011-15021 (2007)

22. A. Iadicicco et al., "Self temperature referenced refractive index sensor by non-uniform thinned fiber Bragg gratings," Sens. Actuators B Chem. 120(1), 231-237 (2006).

23. Y. J. Rao et al., "Optical in-fiber Bragg grating sensor system for medical applications," J. Biomed. Opt. 3(1), 38-44 (1998).

24. S. Silvestri and E. Schena, "Optical-fiber measurement systems for medical applications," Optoelectronics Device and Applications, P. Predeep, Ed., InTech, Croatia (2011).

25. A. F. Omar, "Fiber optic sensors: an introduction for engineers and scientists," Sens. Rev. 33(2) (2013).

26. Q. Yu et al., "Study on optical fiber Bragg grating temperature sensors for human body temperature monitoring," in IEEE Symp. Photonics and Optoelectronics, IEEE (2012).

27. D. Tosi et al., "Fiber-optic combined FPI/FBG sensors for monitoring of radiofrequency thermal ablation of liver tumors: ex vivo experiments," Appl. Opt. 53, 2136 (2014).

28. D. Polito et al., "Development and characterization of a fibre Bragg grating temperature probe for medical laser ablation therapy," Proc. IEEE Sens. 2014, 1134-1137 (2014).

29. D. Tosi, E. G. Macchi, and A. Cigada, "Fiber-optic temperature and pressure sensors applied to radiofrequency thermal ablation in liver phantom: methodology and experimental measurements," J. Sens. 2015(1), 909012 (2015).

30. D. Tosi et al., "Fiber-optic chirped FBG for distributed thermal monitoring of ex-vivo radiofrequency ablation of liver," Biomed. Opt. Express 5(6), 1799 (2014).

31. D. Tosi et al., "Distributed fiber-optic sensors for thermal monitoring in radiofrequency thermal ablation in porcine phantom," Proc. IEEE Sens., 2014, 39-42 (2014).
32. P. Saccomandi et al., "Temperature monitoring during microwave ablation in ex vivo porcine livers," Eur. J. Surg. Oncol. 41(12), 1699-1705 (2015)

33. G. Palumbo et al., "Fiber Bragg gratings for temperature monitoring during medical radiofrequency treatments," in Accept. to Eurosensors Conf. (2016).

34. A. D. Kersey et al., "Fiber grating sensors," J. Lightwave Technol. 15(8), 1442-1463 (1997).

35. K. O. Hill and G. Meltz, "Fiber Bragg grating technology fundamentals and overview," J. Lightwave Technol. 15(8), 1263-1276 (1997).

36. B. A. Tahir, J. Ali, and R. Abdul Rahman, "Fiber Bragg Grating based system for temperature measurements," Int. J. Mod. Phys. B 23(10), 2349-2356 (2009)

37. M. Pai et al., "Liver resection with bipolar radiofrequency device: Habib 4X," HPB 10(4), 256-260 (2008).

38. M. Pai et al., "Use of bipolar radiofrequency in parenchymal transection of the liver, pancreas and kidney," Dig. Surg. 29(1), 43-47 (2012).

39. "User's guide and service manual RITA 1500X" pp. 19-23, AngioDynamics Inc.

40. E. G. Macchi et al., "Temperature distribution during RF ablation on ex vivo liver tissue: IR measurements and simulations," Heat Mass Transfer 51(5), 611-620 (2015).

Giovanna Palumbo received her MSc degree in biomedical engineering from the University of Naples "Federico II," Naples, Italy, in 2012. She is currently pursuing the $\mathrm{PhD}$ in information engineering with a focus on fiber optic sensors with the University of Naples "Parthenope," Naples. She joined the Department of Engineering, University of Naples "Parthenope," in 2014.

Agostino ladicicco is associate professor at the Department of Engineering, University of Naples Parthenope, Italy. He received his electronic engineering (cum laude) degree from the Second University of Naples, Naples, Italy, and his PhD in information engineering from the University of Sannio, Benevento, Italy, in 2002 and 2005 , respectively. His research activity has been focused on optoelectronics and photonics devices for sensing and communications applications since 2002.

Daniele Tosi is assistant professor at Nazarbayev University, School of Engineering. He received his BEng, MEng in telecommunication engineering, and $\mathrm{PhD}$ degrees in electronic engineering from Politecnico di Torino in 2004, 2006, and 2010, respectively. His research activity includes fiber-optic sensors, fiber Bragg gratings, biosensors, and biomedical sensor applications.

Paolo Verze is assistant professor at the Department of Urology of the University of Naples "Federico II," School of Medicine and Surgery Naples, Italy. His specialty areas include uro-oncology, sexual medicine and laparoscopic and robotic surgery. He is on the Guidelines Committee for the European Association of Urology, is the author of over 35 original articles and is a reviewer for European Urology, The Journal of Urology, and the British Journal of Urology International.

Juliet Ippolito, BA Vassar College, MPhil University of Dundee, is a translator, editor and medical communications specialist at the Urology Faculty of the University of Naples School of Medicine and Surgery "Federico II." She is also an English language lecturer at the University of Naples "L'Orientale." She is the former scientific secretary for the European Federation of Corrosion and has a number of publications in the optical, biomedical and materials engineering fields.

Stefania Campopiano is associate professor of electronics and optoelectronics at the University of Naples "Parthenope," Italy. She received her Electronic Engineering degree (cum laude) at the University of Naples Federico II, Italy, and her PhD in electronic engineering from the Second University of Naples, Italy, in 1999 and 2003 , respectively. Since 1999, her research activity is focused on the field of optoelectronic devices for sensing and telecommunication applications.

Biographies for the other authors are not available. 